\title{
Loss of the deubiquitinase OTULIN promotes hepatocellular carcinoma (HCC) in an mTOR-dependent manner
}

\author{
Antonio Marzio ${ }^{1,2} \cdot$ Michele Pagano $^{1,2,3}$ \\ Received: 10 March 2020 / Accepted: 10 March 2020 / Published online: 30 March 2020 \\ (c) The Author(s), under exclusive licence to ADMC Associazione Differenziamento e Morte Cellulare 2020
}

Liver cancer is the second most frequent cause of cancerrelated death worldwide [1]. Chronic liver inflammation as well as metabolic alterations have been shown to play an important role in the pathogenesis of hepatocellular carcinoma (HCC), the most common type of liver tumor. However, the mechanistic link between chronic liver inflammation and HCC development is still poorly understood. Posttranslational modification of proteins by ubiquitin $(\mathrm{Ub})$ conjugation plays an important role in a range of cellular processes, including cell division, DNA damage, signal transduction, inflammation, and cancer development $[2,3]$. Nondegradative ubiquitination signaling is a key mechanism in the regulation of both inflammatory and immune responses via NF-kB activation [4]. Among the various nondegradative Ub linkages that have been described, Ub chains linked via methionine 1 (Met1-polyUb) have been shown to participate in the regulation of the innate immune response. These chains are assembled by the linear Ub chain assembly complex (LUBAC) composed of HOIP, HOIL-1, and SHARPIN1 [5, 6].

Previous studies have revealed that OTULIN (OTU family deubiquitinase with linear linkage specificity) exclusively hydrolyzes M1-polyUb from proteins that have been modified by LUBAC, and is critical for preventing both cell death and a severe autoinflammatory disease, named OTULIN-related autoinflammatory syndrome $[5,7,8]$. However, despite a clear involvement of OTULIN in the

Michele Pagano

michele.pagano@nyulangone.org

1 Department of Biochemistry and Molecular Pharmacology, New York University School of Medicine, New York, NY 10016, USA

2 Perlmutter Cancer Center, New York University School of Medicine, New York, NY 10016, USA

3 Howard Hughes Medical Institute, New York University School of Medicine, New York, NY 10016, USA regulation of innate immunity, its potential involvement in cancer development had not been investigated.

Damgaard et al. [9] now reveal that liver OTULINdeficiency causes both spontaneous steatohepatitis and extensive fibrosis promoting the development of HCC. The study shows that hepatocyte-specific deletion of OTULIN induces premalignant tumors by 8 weeks of age followed by HCC progression within 1 year. Furthermore, progression to HCC in OTULIN-deficient mice was concomitant with metabolic alterations, apoptosis, and inflammation in the liver.

Given the established role of OTULIN in immune cells [10], Damgaard et al. developed several mouse models to dissect the molecular pathways associated with HCC progression and better understand the role of OTULIN in nonimmune cell types. To assess the nonimmune cell functions of OTULIN, irradiated OTULIN null mice were reconstituted with wild-type bone marrow to generate chimeras with immune cells carrying wild-type OTULIN. Phenotypic analysis of these mice revealed severe weight loss accompanied by liver failure that was characterized by (1) enhanced liver damage; (2) increased blood neutrophils; (3) histological signs of acute hepatitis; and (4) increased $\mathrm{M} 1-$ polyUb conjugates in Otulin-KO $\mathrm{K}^{\text {chim }}$ mice.

Analysis of mice with a hepatocyte-specific deletion of OTULIN revealed the presence of severe liver disease with macroscopic lesions and dysplastic nodules. Furthermore, OTULIN-deficient livers showed steatosis, hyperplasia, and signs of inflammation, highlighting the cytoprotective function of OTULIN in the liver. This unexpected observation not only underscores the importance of OTULIN in the regulation of M1-polyUB signaling in liver cells, but also expands the range of pathologies associated with OTULIN-deficiency.

It is established that systemic inflammation caused by OTULIN-deficiency is a result of increased NF- $\mathrm{KB}$ signaling, which can be ameliorated by blocking TNF $\alpha$ signaling [7, 10]. Intriguingly, Damgaard et al. found that deletion of TNFR1 had no effect on the liver defects of 
OTULIN-deficient mice, indicating that alternative mechanisms can drive the liver pathology in these mice.

Phenotypic analysis of livers from early postnatal OTULIN-deficient mice revealed dramatic changes in expression of mTOR regulators, such as the TSC complex and Rheb, accompanied by an aberrant activation of the mTOR pathway. Interestingly, rapamycin was able to rescue the liver pathologies associated with OTULIN-deficiency, indicating a central role for OTULIN in the regulation of mTOR signaling in the liver. These findings not only expand the function of OTULIN beyond the immune compartment and demonstrate a bone fide tumor suppressor function for this deubiquitinase, but also provide the first mechanistic link between M1-polyUb and the mTOR pathway.

A number of intriguing questions arise from the findings by Damgaard et al. [9]. For example, how does accumulation of Met1-polyUb in OTULIN-ablated livers contribute to the activation of mTOR pathway? Is Met1-polyUb directly conjugated to some proteins regulating the mTOR pathway? And finally, does OTULIN counteracts an Ub ligase that targets key regulators of the mTOR signaling using Met1-polyUb?

The work described here shows that the Ub signaling, through polyubiquitin-linkage specificity, regulates key cellular processes and is even more complex than previously assumed. Importantly, this manuscript reminds us that polyubiquitin-linkage specificity dictates signaling events, and that its deregulation in cancer cells needs to be investigated in detail.
Publisher's note Springer Nature remains neutral with regard to jurisdictional claims in published maps and institutional affiliations.

\section{References}

1. Llovet JM, Zucman-Rossi J, Pikarsky E, Sangro B, Schwartz M, Sherman M, et al. Hepatocellular carcinoma. Nat Rev Dis Prim. 2016;2:16018.

2. Oh E, Akopian D, Rape M. Principles of ubiquitin-dependent signaling. Annu Rev Cell Dev Biol. 2018;34:137-62.

3. Senft D, Qi J, Ronai ZA. Ubiquitin ligases in oncogenic transformation and cancer therapy. Nat Rev Cancer. 2018;18:69-88.

4. $\mathrm{Hu} \mathrm{H}$, Sun SC. Ubiquitin signaling in immune responses. Cell Res. 2016;26:457-83.

5. Hrdinka M, Gyrd-Hansen M. The Met1-linked ubiquitin machinery: emerging themes of (de)regulation. Mol Cell. 2017;68: $265-80$.

6. Shimizu Y, Taraborrelli L, Walczak H. Linear ubiquitination in immunity. Immunol Rev. 2015;266:190-207.

7. Damgaard RB, Elliott PR, Swatek KN, Maher ER, Stepensky P, Elpeleg O, et al. OTULIN deficiency in ORAS causes cell typespecific LUBAC degradation, dysregulated TNF signalling and cell death. EMBO Mol Med. 2019;11:e9324. https://doi.org/10. 15252/emmm.201809324.

8. Keusekotten K, Elliott PR, Glockner L, Fiil BK, Damgaard RB, Kulathu Y, et al. OTULIN antagonizes LUBAC signaling by specifically hydrolyzing Met1-linked polyubiquitin. Cell. 2013;153:1312-26.

9. Damgaard RB, Jolin HE, Allison MED, Davies SE, McKenzie ANJ, Komander D. OTULIN protects the liver against cell death, inflammation, fibrosis, and cancer. Cell Death Differ. 2020.

10. Damgaard RB, Walker JA, Marco-Casanova P, Morgan NV, Titheradge HL, Elliott PR, et al. The deubiquitinase OTULIN is an essential negative regulator of inflammation and autoimmunity. Cell. 2016;166:1215-30.e1220. 